DOI: 10.1002/anie.200501906

\section{Mesoscopic Metallosupramolecular Texturing by Hierarchic Assembly**}

\author{
Sylvain Clair, Stéphane Pons, Harald Brune, Klaus Kern, \\ and Johannes V. Barth*
}

Currently, there is a strong interest in improving the fabrication and mesoscopic integration of functional nanosystems at surfaces. In particular, new pathways need to be developed to provide methodologies for the synthesis and embedding of nanostructures across multiple length scales. ${ }^{[1-4]}$ Herein, we introduce the combination of nanopatterning and controlled metal-organic assembly to process prestructured metallic templates and generate arrangements at a higher hierarchical level. We present results of scanning tunneling microscopy (STM) on the metal-directed assembly and mesoscopic organization of supramolecular architectures by using a textured metal substrate, that is, an $\mathrm{Au}(111)$ surface decorated with $\mathrm{Fe}$ or Co nanodot arrays. By tuning the local reaction conditions with co-deposited dicarboxylate linker molecules, distinct low-dimensional metallosupramolecular systems have been synthesized, which include regularly spaced iron terephthalate ribbons that form a grating whose extension is only limited by the substrate terrace morphology. These findings indicate that hierarchic assembly protocols blending physical nanopatterning ${ }^{[5-9]}$ and supramolecular engineering ${ }^{[10-13]}$ on surfaces are generally useful for fabricating nanomaterials with control of features at the mesoscopic level.

Metal-directed self-assembly of functional molecules provides a versatile strategy towards highly organized supramolecular systems. ${ }^{[14-17]}$ Recent findings reveal that similar processes can be conducted at surfaces, where notably a series of metal-carboxylate architectures were generated, including mononuclear arrays, ladder structures, and nanoporous 2D metal-organic coordination networks. ${ }^{[13,18-21]}$ However, until now these systems have been constructed on homogeneous substrates on which typically organic layers were exposed to a

[*] Dr. S. Clair, Dr. S. Pons, Prof. H. Brune, Prof. K. Kern, Prof. J. V. Barth Institut de Physique des Nanostructures

Ecole Polytechnique Fédérale de Lausanne

1015 Lausanne (Switzerland)

E-mail: jvb@chem.ubc.ca

Prof. K. Kern

Max-Planck-Institut für Festkörperforschung

Heisenbergstrasse 1, 70569 Stuttgart (Germany)

Prof. J. V. Barth

Departments of Chemistry and Physics \& Astronomy

The University of British Columbia

Vancouver, B.C. V6T1Z4 (Canada)

[***] This work was supported by the Swiss National Science Foundation (as part of the European Science Foundation EUROCORES Programme SONS) and the EC Sixth Framework Programme (as part of the STREP BiOMACH). flux of metal atoms. To control the spatial distribution of surface coordination architectures, it is promising to explore prestructured surfaces, which-due to their intrinsic anisotropy, local concentration of reactants and site reactivityallow us to direct and confine the assembly. This control is not possible with homogeneous substrates. Herein we present a temperature-controlled STM investigation of the steering of metallosupramolecular reticulation by using nanopatterned templates. This approach is a model for a two-stage assembly that combines self-organized metal-on-metal growth followed by chemical processing. This methodology may allow the fabrication of low-dimensional functional nanosystems, steer the formation of (metallo-) supramolecular assemblies, enable the study and control of metal-molecule interfacing, or structure template layers in the $100 \mathrm{~nm}$ regime for handling nanoscale objects.

Specifically, we employed regular lattices of transition metal dots by depositing small amounts of cobalt or iron on $\mathrm{Au}(111)$ at room temperature. Due to preferred nucleation of metal islands at the elbow sites of a reconstructed surface, ${ }^{[22]}$ $\mathrm{Fe}$ or Co nanoarrays are readily obtained. ${ }^{[23,24]}$ Islands of monoatomic height evolve as a consequence of Fe deposition, whereas bilayer dots are encountered with Co deposition. In agreement with earlier findings, the STM data confirm that the $\mathrm{Au}(111)$ reconstruction is maintained as demonstrated in the exemplary results in Figure 1. The superstructure is equivalent for the two elements. The lattice unit cell is nearly rectangular and ca. $15 \mathrm{~nm} \times 7 \mathrm{~nm}$ in size. However, on smaller terraces and in the vicinity of defects substantial variations can occur. Typically $0.1 \mathrm{ML}$ of $\mathrm{Co}$ or Fe atoms produces islands of about 140 atoms (one ML of metal atoms refers to a hypothetical perfect commensurate monatomic layer on the gold surface). As we will show, these islands act as nanoreservoirs for the metal-directed assembly of co-deposited terephthalic acid molecules (TPA, 1,4-benzenedicarboxylic acid).

While the TPA molecules are entirely preserved on the bare $\mathrm{Au}(111)$ substrate, where they form hydrogen-bonded sheets, ${ }^{[25]}$ they readily react with an available Co or Fe atom at room temperature or at slightly elevated temperatures to form metal-organic nanostructures. The respective complexation is a reaction between the carboxyl groups of the molecules and one or more transition metal adatoms (M), which eventually can be extracted from the islands. This implies deprotonation of the carboxyl group, that is, an Mcarboxylate evolves and leads to the formation of reticulated planar metal-organic arrangements. The resulting hydrogen atoms are expected to recombine to form molecular hydrogen, which is known to thermally desorb from noble-metal substrates under the conditions employed. ${ }^{[26]}$ The complexation reaction is therefore irreversible and can be represented formally by the following net equation [Eq.(1)]:

$2 \mathrm{RCOOH}+\mathrm{M} \rightarrow(\mathrm{RCOO})_{2} \cdots \mathrm{M}+\mathrm{H}_{2}(\uparrow)$

Since the diffusivity of the complexes is limited and the metal-organic bond has appreciable strength, the connection between building blocks can be kept localized in the vicinity of the originally deposited metal dots. In contrast, the high 

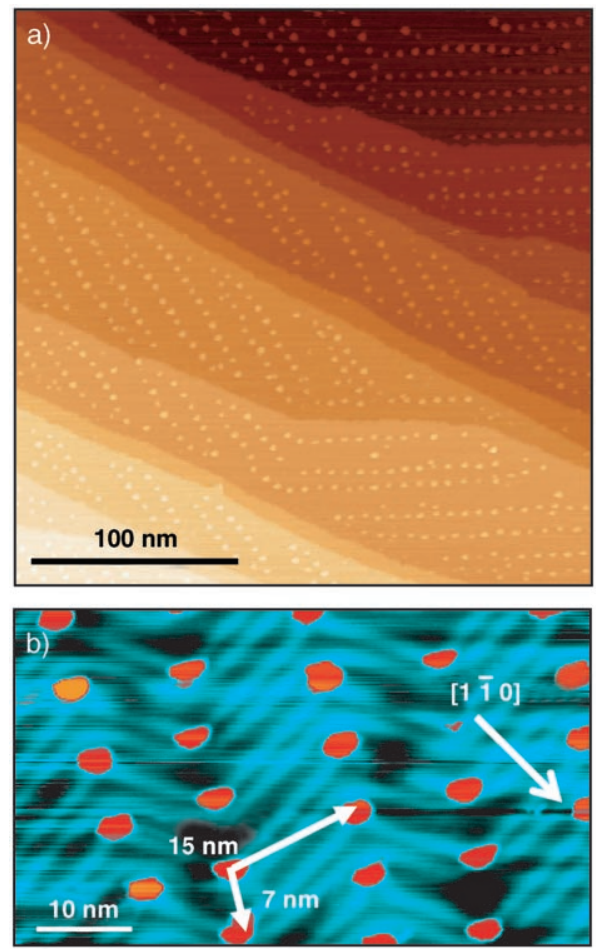

Figure 1. STM images of the $\mathrm{Au}(111)$ template with its chevron reconstruction dislocation pattern decorated with transition-metal arrays, which reflect preferential nucleation at elbow sites. a) Bilayer cobalt islands form regular lattices on several terraces $(0.14 \mathrm{ML}$ Co deposited at room temperature; image size $280 \times 280 \mathrm{~nm}^{2}$,

$V=-25 \mathrm{mV}, I=0.8 \mathrm{nA}$, recorded at room temperature). b) Monolayer high $\mathrm{Fe}$ dots (red spots) on a single terrace with the characteristic double lines of the reconstruction simultaneously resolved. The unit cell of the regular lattice is ca. $15 \mathrm{~nm} \times 7 \mathrm{~nm}$ in size. $(0.1 \mathrm{ML} \mathrm{Fe}$ deposited at room temperature; image size $60 \times 35 \mathrm{~nm}^{2}, V=20 \mathrm{mV}$, $I=1 \mathrm{nA}$, recorded at $5 \mathrm{~K}$ ).

mobility of TPA on bare $\mathrm{Au}(111)$ provides the necessary easy transport of the incoming molecule flux to the rims of the reactive metal islands.

Careful tuning of the conditions for molecular deposition has been used to steer the formation of various metalcarboxylate arrangements. In particular we have obtained architectures comprising regularly spaced nanoporous islands, extended metallosupramolecular ribbons, or one-dimensional systems. In general we observed that use of cobalt gives rise to better-ordered structures at the molecular scale than iron, while the latter allows easier steering at the mesoscopic level. The set of parameters that we optimized to obtain wellordered architectures are the dosage (experiments were performed in the sub-monolayer range), the relative stoichiometry of M:TPA, the thermal energy of the adspecies tuned by the temperature of the substrate during deposition, and postdeposition annealing. With adequate temperature control, it is possible to obtain surface nanostructures comprising a) exclusively M-TPA compounds $(\mathrm{M}: \mathrm{TPA}<1)$ or b) coexisting residual metal nanodots and reticulated M-TPA compounds $(\mathrm{M}:$ TPA $>1)$.

Figure 2a shows an STM image of an Fe nanoarray on $\mathrm{Au}(111)$ after exposure to TPA with a stoichiometry of about

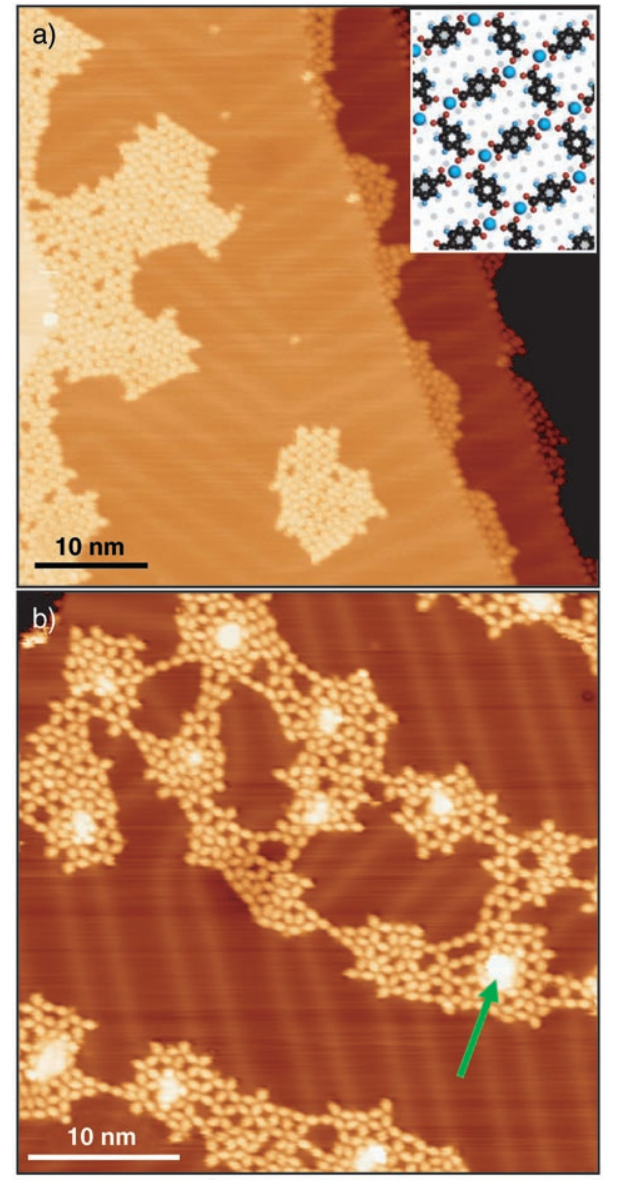

Figure 2. Controlling the Fe-TPA complexation reaction. a) Complete dissolution of Fe islands for a Fe-TPA stoichiometry of ca. 1:1 following deposition of TPA on the Fe nanoarrays at $355 \mathrm{~K}$. Fecarboxylate islands preferentially decorate step edges (image size $51 \times 51 \mathrm{~nm}^{2}, V=-2.5 \mathrm{~V}, I=0.5 \mathrm{nA}$, recorded at $5 \mathrm{~K}$ ). The inset shows a tentative model for an ideal Fe-TPA nanogrid where at each diiron center the endgroups of four molecular linkers come together. b) Localized reaction with TPA deposition at $325 \mathrm{~K}$ and 10 min postdeposition annealing at $330 \mathrm{~K}$. The Fe islands are only partially consumed in the complexation reaction. The resulting nanogrid structure reflects selective growth of metal-organic islands at residual Fe dots (Fe island indicated by arrow; $0.1 \mathrm{ML} \mathrm{Fe}, 0.25 \mathrm{MLTPA}$; image size $38 \times 38 \mathrm{~nm}^{2}, V=1.5 \mathrm{~V}, I=0.2 \mathrm{nA}$, recorded at $5 \mathrm{~K}$ ).

one Fe atom per TPA molecule; the sample was maintained at $T=355 \mathrm{~K}$ during the molecular deposition. This procedure resulted in the complete dissolution of the $\mathrm{Fe}$ dots and the formation of irregular islands that coexist with the unperturbed chevron pattern of the $\mathrm{Au}(111)$ substrate. While the presence of the latter clearly indicates that surface alloying between $\mathrm{Fe}$ and the substrate does not occur in the terraces, the irregular islands are clearly distinct from the extended close-packed quasi-hexagonal domains of hydrogen-bonded molecules that is characteristic of the adsorption of pure TPA. ${ }^{[25]}$ The islands have open networks, which are reminiscent of the ordering found in nanoporous iron-carboxylate layers with TPA and related ditopic linear linkers on a square $\mathrm{Cu}(100)$ substrate. ${ }^{[13,18,28]}$ Thus we conclude that these islands consist of iron-diterephthalate arrangements stabilized by metal-organic reticulation. The size of the characteristic open 
nanogrids is about $1 \times 1.2 \mathrm{~nm}^{2}$; their relation to the metalorganic networks on $\mathrm{Cu}(100)$ suggests that, similarly, the network nodes consist of diiron centers surrounded by four molecules. ${ }^{[13,18,28]}$ This analogy is illustrated in the tentative model for an ideal Fe-TPA nanogrid with 1:1 stoichiometry depicted in the inset in Figure 2 a. However, in contrast to the square $\mathrm{Cu}(100)$ substrate, the metal centers are not directly resolved by STM and the quasi-hexagonal lattice of $\mathrm{Au}(111)$ offers no match with an overlayer structure composed of rectangular motifs. As a consequence the Fe-TPA islands do not exceed 10 to $15 \mathrm{~nm}$ in size and present frequent distortions and misorientations. Indeed, we found that it is impossible to create extended regular nanogrid domains despite the smoothness of the substrate, which indicates that while the strength of the metal-ligand interactions is appreciable, it is not sufficient to dictate the entire overlayer structure. In contrast with $\mathrm{Co} / \mathrm{Au}(111)$ nanotemplates, the Fe-TPA system is more sensitive to stoichiometry and temperature and is harder to control. While Co-TPA complexes organize in locally regular porous islands for the deposition parameters described above, Fe-TPA complex islands are rather disordered and give rise to a mixture of regular metal-organic grids and complicated irregular phases. Finally, the decoration of $\mathrm{Au}$ step edges is associated with local intermixing or coupling of $\mathrm{Au}$ and $\mathrm{Fe}$, which is in agreement with findings for a pure $\mathrm{Fe} / \mathrm{Au}(111)$ system, ${ }^{[29]}$ and implies that $\mathrm{Fe}$ atoms can transport under these reaction conditions either directly by evaporation from the Fe dots or indirectly by release in the form of TPA-Fe complexes. In general, for Fe-TPA assemblies with 1:1 stoichiometry (see for example Figure $2 \mathrm{a}$ ), step decoration occurs preferentially at the bottom edges.

By lowering the annealing or deposition temperature and reducing the TPA concentration, we succeeded in confining the complexation reaction. This confinement is demonstrated in the STM image of Figure 2b, which shows the result of a TPA dosage too small to form networks with all of the Fe atoms available on the surface. Consequently, there is merely a partial dissolution of Fe islands and the cores of the metal islands remain at the $\mathrm{Au}(111)$ elbow sites. These residual Fe dots act as anchoring sites for reticulated metal-organic assemblies. All Fe islands are decorated by nanoporous irregular islands and chainlike features where the endgroups of the rodlike molecules are connected. The formation of these open structures again is in direct contrast with the compact packing in hydrogen-bonded pure TPA layers. ${ }^{[25]}$ Consequently these arrangements must be stabilized by the incorporation of metal centers and reflect the limited surface mass transport of the reactants under the conditions employed. Thus the Fe-TPA nanogrid structure is poorly developed but the metal-organic islands coalesce and local bridges have evolved between them.

The connection between the localized complex islands is favored along the symmetry axis of the chevron pattern along the elbows. Two aspects can explain this growth anisotropy. First, along this direction is the shortest distance between two metallic dots and, indeed, we observed that the closer two metal-organic islands are, the more likely they are to coalesce. Second, the substrate reconstruction pattern with its substantial lattice distortions at the elbows may induce directional molecular transport and orientations. By carefully controlling the molecular surface concentration, extended metallosupramolecular ribbons with regular residual $\mathrm{Fe}$ anchoring sites can be fabricated. This point is illustrated in the STM image of Figure 3, which shows the mesoscopic

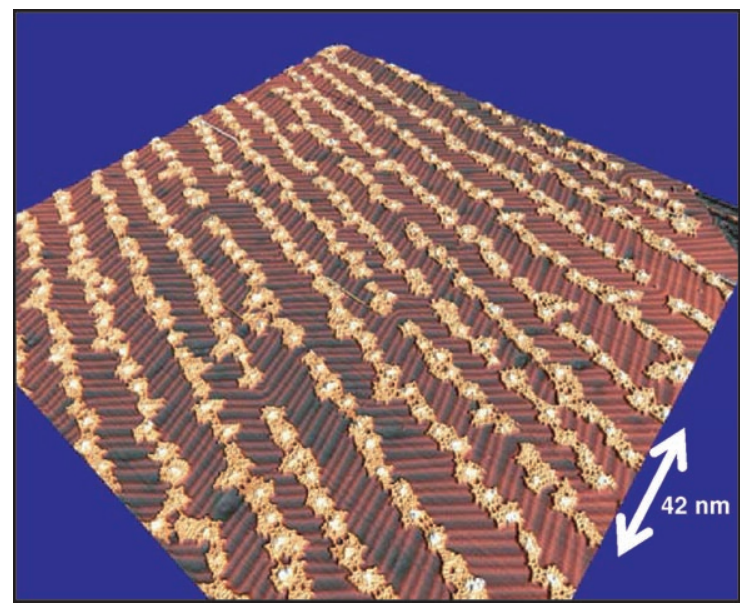

Figure 3. Mesoscopic ordering of metallosupramolecular ribbons. Feterephthalate linkages evolve between next-neighbor Fe islands following TPA exposure. The residual Fe dots at the elbow sites of the reconstruction anchor the pattern, that is, the spatial distribution of the Fe dots steers the organization. The length of the metal-organic ribbons is around several hundred $\mathrm{nm}$, with typical widths of 5-10 nm The respective aspect ratio of the stripes is commonly greater than 20 to 40 (image size $220 \times 220 \mathrm{~nm}^{2}, V=-1.5 \mathrm{~V}, I=0.8 \mathrm{nA}$, recorded at $5 \mathrm{~K} ; 0.1 \mathrm{ML}$ Fe exposed to $0.25 \mathrm{ML}$ TPA at $325 \mathrm{~K}$, i.e., fourfold Fe excess with respect to ideal 1:1 nanogrid stoichiometry).

ordering of Fe-TPA ribbons induced by the chevron structure. The widths of the stripes in this architecture are usually around 5 to $10 \mathrm{~nm}$, while their lengths can reach hundreds of nanometers, that is, they span entire terraces until they terminate at the Au step edges. Therefore, the aspect ratios of the ribbons frequently exceeds 40 and by using vicinal transition-metal decorated $\mathrm{Au}(111)$ substrates with parallel step edges, ${ }^{[7]}$ macroscopically elongated metal-organic ribbons are expected to form.

By further fine tuning the deposition parameters, we assembled monomolecular chains in the Co-TPA system on $\mathrm{Au}(111)$ as shown in Figure 4. Such metal-organic wires form at reduced molecular coverages but with limited success, and these 1D structures coexist with nanogrid patches (not shown). The wires establish a connection between two adjacent dots and therefore do not exceed $10 \mathrm{~nm}$ in length. The exact internal structure and stoichiometry of these chains composed of M-terephthalate segments could not be determined. However, the distance between the molecular building blocks in the chain increases by about $10 \%$ with respect to that in the hydrogen-bonded assemblies, which corroborates that it arises from a complexation of TPA and Co atoms, where distances of $0.2 \mathrm{~nm}$ between metal center and carboxylate oxygen atoms are expected. Moreover, the 1D Co-TPA chains anchored by the Co dots are stable at room temperature, which reflects the appreciable strength of metal-ligand 


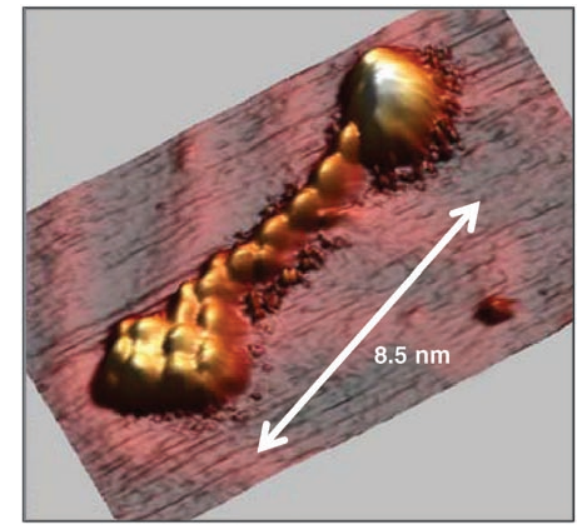

Figure 4. Monomolecular Co-TPA "wire" linking two cobalt nanodots. The rims of the Co islands are also decorated with molecules. This 1D structure coexists on the surface with regions covered by nanogrids (doses: $0.2 \mathrm{ML} \mathrm{Co,} \mathrm{0.2} \mathrm{ML} \mathrm{TPA;} \mathrm{sample} \mathrm{kept} \mathrm{at} 360 \mathrm{~K}$ during TPA deposition; image size $15 \times 8 \mathrm{~nm}^{2}, V=-700 \mathrm{mV}, I=0.6 \mathrm{nA}$, recorded at room temperature).

interactions that exceed hydrogen-bonding interactions of 1D TPA polymers. Accordingly, monomolecular hydrogenbonded chains can neither be obtained by molecular selfassembly at room temperature ${ }^{[25]}$ nor are they encountered under cryogenic conditions.

In conclusion we have demonstrated that substrates patterned with transition-metal nanoarrays can be processed by tuning and spatially confining their reaction with functional molecular species. Methodologies that use such hierarchic assembly protocols are conceivable for a great variety of systems and can be applied at substrates with different symmetries, physical, and chemical properties. Thus, they are a promising route for the metallo-supramolecular engineering of patterned templates by using linker molecules with specific functionalities. We expect that these findings will contribute to an improved control of matter at the nanoscale, the mesoscopic organization of template structures, and the design of supramolecular functional systems.

\section{Experimental Section}

Experiments were performed in a ultrahigh vacuum system (base pressure $<3 \times 10^{-10}$ mbar) comprising a home-built STM working at variable temperature $(400-5 \mathrm{~K})$ as previously described. ${ }^{[27]}$ Data were recorded in constant-current mode; indicated voltages refer to the sample bias. The $\mathrm{Au}(111)$ substrate was prepared by repeated cycles of room-temperature Ar bombardment $\left(\approx 5 \mu \mathrm{A} \mathrm{cm}^{-2}\right.$ at $\left.800 \mathrm{eV}\right)$ then annealing at $900 \mathrm{~K}$. Terraces larger than $100 \mathrm{~nm}$ were commonly observed. Iron and cobalt were evaporated at low deposition rates $(<1 / 3$ ML per minute). TPA molecules (FLUKA, purity $>99 \%$ ) were evaporated with an organic molecular beam deposition (OMBD) source, by heating a crucible to $150^{\circ} \mathrm{C}$ - the pertaining evaporation rate was $\approx 1 \mathrm{ML}$ (i.e., a complete monomolecular layer) per minute. ${ }^{[27]}$ A metal-to-TPA stoichiometry of 1:1 corresponds to a coverage ratio of $\approx 9: 1$.

Received: June 2, 2005

Revised: August 24, 2005

Published online: October 17, 2005
Keywords: coordination modes - nanotechnology · scanning probe microscopy · self-assembly · supramolecular chemistry

[1] T. Ogino, H. Hibino, T. Homma, Y. Kobayashi, K. Prabhakaran, K. Sumitomo, H. Omi, Acc. Chem. Res. 1999, 32, 447.

[2] Y. N. Xia, J. A. Rogers, K. E. Paul, G. M. Whitesides, Chem. Rev. 1999, 99, 1823.

[3] H. Cölfen, S. Mann, Angew. Chem. 2003, 115, 2452; Angew. Chem. Int. Ed. 2003, 42, 2350.

[4] C. Lin, C. R. Kagan, J. Am. Chem. Soc. 2003, 125, 336.

[5] K. Kern, H. Niehus, A. Schatz, P. Zeppenfeld, J. George, G. Comsa, Phys. Rev. Lett. 1991, 67, 855.

[6] H. Brune, M. Giovannini, K. Bromann, K. Kern, Nature 1998, 394, 451.

[7] V. Repain, G. Baudot, H. Ellmer, S. Rousset, Europhys. Lett. 2002, 58, 730 .

[8] M. Corso, W. Auwärter, M. Muntwiler, A. Tamai, T. Greber, J. Osterwalder, Science 2004, 303, 217.

[9] R. Otero, Y. Naitoh, F. Rosei, P. Jiang, P. Thostrup, A. Gourdon, E. Laegsgaard, I. Stensgaard, C. Joachim, F. Besenbacher, Angew. Chem. 2004, 116, 2144; Angew. Chem. Int. Ed. 2004, 43, 2092.

[10] J. V. Barth, J. Weckesser, C. Cai, P. Günter, L. Bürgi, O. Jeandupeux, K. Kern, Angew. Chem. 2000, 112, 1285; Angew. Chem. Int. Ed. 2000, 39, 1230.

[11] J. A. Theobald, N. S. Oxtoby, M. A. Phillips, N. R. Champness, P. H. Beton, Nature 2003, 424, 1029.

[12] T. Yokoyama, S. Yokoyama, T. Kamikado, Y. Okuno, S. Mashiko, Nature 2001, 413, 619.

[13] S. Stepanow, M. Lingenfelder, A. Dmitriev, H. Spillmann, E. Delvigne, N. Lin, X. Deng, C. Cai, J. V. Barth, K. Kern, Nat. Mater. 2004, 3, 229.

[14] D. Philp, J. F. Stoddart, Angew. Chem. 1996, 108, 1242; Angew. Chem. Int. Ed. Engl. 1996, 35, 1154.

[15] S. Leininger, B. Olenyuk, P. J. Stang, Chem. Rev. 2000, 100, 853.

[16] J. M. Lehn, Proc. Natl. Acad. Sci. USA 2002, 99, 4763.

[17] B. J. Holliday, C. A. Mirkin, Angew. Chem. 2001, 113, 2076; Angew. Chem. Int. Ed. 2001, 40, 2022.

[18] A. Dmitriev, H. Spillmann, N. Lin, J. V. Barth, K. Kern, Angew. Chem. 2003, 115, 2774; Angew. Chem. Int. Ed. 2003, 42, 2670.

[19] J. V. Barth, J. Weckesser, N. Lin, S. Dmitriev, K. Kern, Appl. Phys. A 2003, 76, 645.

[20] H. Spillmann, A. Dmitriev, N. Lin, P. Messina, J. V. Barth, K. Kern, J. Am. Chem. Soc. 2003, 125, 10725.

[21] A. Dmitriev, H. Spillmann, M. Lingenfelder, N. Lin, J. V. Barth, K. Kern, Langmuir 2004, 41, 4799.

[22] J. V. Barth, H. Brune, G. Ertl, R. J. Behm, Phys. Rev. B 1990, 42, 9307.

[23] B. Voigtländer, G. Meyer, N. M. Amer, Phys. Rev. B 1991, 44, 10354.

[24] J. A. Stroscio, D. T. Pierce, R. A. Dragoset, P. N. First, J. Vac. Sci. Technol. A 1992, 10, 1981.

[25] S. Clair, S. Pons, A. P. Seitsonen, H. Brune, K. Kern, J. V. Barth, J. Phys. Chem. B 2004, 108, 14585.

[26] X.-L. Zhou, J. M. White, B. E. Koel, Surf. Sci. 1989, 218, 201.

[27] S. Clair, PhD thesis, EPF (Lausanne), 2004.

[28] M. A. Lingenfelder, H. Spillmann, A. Dmitriev, S. Stepanov, N. Lin, J. V. Barth, K. Kern, Chem. Eur. J. 2004, 10, 1913.

[29] S. Shiraki, H. Fujisawa, M. Nantoh, M. Kawai, J. Electron Spectrosc. Relat. Phenom. 2004, 137-140, 177. 